\title{
Magnetic resonance imaging based muscle strain rate mapping during eccentric contraction to study effects of unloading induced by unilateral limb suspension
}

\author{
Usha Sinha (1), Vadim Malis (2), Robert Csapo (3), Marco Narici (4), Shantanu Sinha (5)
}

(1) Department of Physics, San Diego State University, San Diego, CA, USA; (2) Department of Physics, University of California San Diego, San Diego, CA, USA;(3) Private University for Health Sciences, Medical Informatics and Technology, ISAG, Research Unit for Orthopedic Sports Medicine and Injury Prevention, Hall, Austria; (4) Institute of Physiology, Department of Biomedical Sciences, University of Padua, Padua, Italy; (5) Department of Radiology, University of California San Diego, San Diego, CA, USA

This article is distributed under the terms of the Creative Commons Attribution Noncommercial License (CC BY-NC 4.0) which permits any noncommercial use, distribution, and reproduction in any medium, provided the original author $(s)$ and source are credited.

\begin{abstract}
Age- and disuse- related loss of muscle force is disproportionately larger than the loss of muscle mass. Earlier studies reported that comparing concentric and eccentric contractions, there is a significant age-related decrease in force only in concentric contractions. Magnetic Resonance Imaging enables mapping of muscle deformation and has been used to study isometric but not eccentric contractions. We report MRI based strain rate mapping of the medial gastrocnemius in subjects pre- and post-unloading induced by Unilateral Limb Suspension. In contrast to isometric contraction, no difference in strain rate indices were observed post-unloading, in conformance with preserved force during eccentric contractions.
\end{abstract}

Key Words: eccentric contraction, strain rate mapping, unilateral limb suspension, velocityencoded phase contrast imaging.

Muscle unloading results in a loss of muscle mass (atrophy) and loss of muscle force output. ${ }^{1}$ Muscle atrophy has its origins in a decrease in protein synthesis that is accompanied by an increase in protein degradation that ultimately leads to a loss in the number and size of muscle fibers. ${ }^{2,3}$ However, most unloading studies have demonstrated that decrease in muscle force far exceeds the decrease in muscle size. This discrepancy remains even after accounting for changes in muscle activation as well as for decreases in specific tension. ${ }^{4,5}$ Force can be transmitted longitudinally and laterally through the adjacent ECM and muscle fibers to the epimysium of the skeletal muscle. ${ }^{6}$ More recently, invasive studies on animal models have identified that force transmission by lateral transmission of force pathways is reduced significantly in very old rodents compared to young rodents. ${ }^{7}$ Further, physiologically derived computational muscle modeling has identified the mechanism by which force is transmitted laterally is through shear strain in the extracellular matrix. ${ }^{8}$ Measurement of shear strain by imaging may allow a non-invasive paradigms. Strain is a measure of tissue deformation with respect to a reference state and requires three-dimensional (3Dal) tissue tracking. Strain Rate describes the rate of regional deformation and does not require 3Dal tracking or a reference state since it is an instantaneous measure. The in-vivo studies on muscle motion using these sequences have been applied to the study of muscle kinematics under isometric or passive plantarflexion. ${ }^{9}$ There have been no strain or SR measurements during eccentric contractions. Eccentric contractions are of interest since many physiological functions occur when the muscle is actively lengthening. Further, eccentric contractions have been shown to be most effective for muscle strengthening. Another interesting observation is that there are significant differences between eccentric and concentric contraction, ${ }^{10}$ with a much larger loss of force generation under concentric contraction than under eccentric contraction, and that age-related reduction in muscle force is not observed during an eccentric contraction. ${ }^{11}$

The Unilateral Limb Suspension (ULLS) model of atrophy and muscle force loss is a well- established model for inducting controlled atrophy. An earlier study reported the results of strain rate analysis on velocity encoded phase contrast MR imaging studies on a cohort 
of subjects pre- and post-unloading induced by ULLS under isometric contraction of the calf muscle. ${ }^{12}$ The latter study found significant changes in the components of the strain rate tensor between pre- and post- unloading conditions; these included changes in the principal strain rate components and in the shear strain rate. Further, loss of muscle force as measured by the maximum voluntary contraction (MVC) was significantly correlated to shear strain rate and to the principal strain in the fiber crosssection in addition to morphological changes. This highlights that the strain rate indices may provide additional determinants of muscle force loss independent of muscle mass (volume). In the current study, the focus is on studying changes in strain rate in the medial gastrocnemius with unloading under submaximal eccentric contractions and comparing it to the earlier report on strain rate indices during isometric contraction (on the same cohort and evaluated in the medial gastrocnemius). These two contraction modes are compared since force production during isometric contraction is reduced by the presence of a stiffer passive matrix while it is enhanced during eccentric contraction allowing exploration of acute atrophy related differences in muscle passive mechanical properties and contractility.

\section{Materials and Methods}

\section{Subjects}

Seven subjects were included in this study after informed consent (approved by Medical Research Ethics Board of University of California San Diego). The criterion for inclusion was that subjects should be moderately active and free from any lower extremity surgery in the six months prior to the study. Muscle atrophy was induced on the (self-reported) non-dominant leg (left for all subjects) with 4-week of chronic unloading using the well-established ULLS model. ${ }^{12}$ The unloading protocol is detailed in Malis V, et al. (2018). ${ }^{12}$

\section{MR imaging}

MR imaging was performed on a 1.5 Tesla Signa HDx MR scanner (GE Medical Systems, Milwaukee, WI), with the subject lying supine, feet first, with left leg in a specially designed foot-pedal device for eccentric contraction (Fig. 1a). The foot pedal device is a MRcompatible, computer-controlled, servo motor driven device, which is capable of rotating the foot through a pre-programmed angular range of motion. The ball of the foot rested on a carbon-fiber plate, onto which an optical pressure transducer (Luna Innovations, Roanoke, VA, USA) was glued, and which could rotate along with the foot. Pressure against the plate was detected by the transducer which was subsequently converted to a voltage and used to trigger the MR image acquisition. The voltage output from the pressure transducer was converted into units of force $(\mathrm{N})$ based on a calibration of the system using disc weights. Images were acquired during submaximal, eccentric contractions, at $35 \%$ of the individual maximum voluntary contraction (MVC). A water saturated fast-spin echo (FSE) sequence was used to identify fascicles in the medial gastrocnemius to choose the optimal orientation for the motion sensitive sequence. The latter scan is based on a Velocity-Encoded Phase Contrast (VE-PC) sequence: oblique sagittal slices (TE: $7.7 \mathrm{~ms}$, TR: $16.4 \mathrm{~ms}$, NEX: 2, FA: $20^{\circ}$, slice thickness: $5 \mathrm{~mm}$, FOV: $30 \mathrm{~cm} \times 22.5 \mathrm{~cm}$ (partial phaseFOV: 0.75$), 256 \times 192$ matrix, 4 views/segment, 5-7 slices, 22 phases (contraction-relaxation cycle of $\sim 3$ $\mathrm{s}), 10 \mathrm{~cm} \cdot \mathrm{s}-13$ directional velocity encoding, 70 repetitions for each slice acquisition.

\section{Image Analysis}

Muscle fascicle end points were identified on the FSE images and fiber orientations were identified at three locations along the MG. The phase images from the VEPC sequence quantify the velocity (vx, vy, vz) at each image voxel. The strain rate $(2 \times 2$ tensor $)$ is calculated from the spatial gradient of the velocity, details of the computation are provided in Reference. ${ }^{12}$ The diagonalization of the SR tensor provides a positive and negative eigenvalue; a positive eigenvalue indicates a local lengthening while a negative eigenvalue indicates a local shortening. Further, the positive and negative eigenvalues are labeled SRfiber that refers to the SR eigenvector with the smaller angle with the muscle fiber direction and SRin-plane that refers to the eigenvalue orthogonal to SRfiber and approximately in the fiber cross-section (Fig. 1b). The third eigenvalue, SRoutplane was inferred from SRfiber and SRin-plane by using muscle incompressibility: SRout-plane $=($ SRfiber + SRin-plane). In addition, the shear strain, SRfc, was computed by rotating the principal axis strain rate tensor by the angle between SRfiber and muscle fiber, denoted as SR-fiber angle.

Region of interest (ROI) measurements were taken in the proximal, middle and distal regions of the MG. The ROIs were tracked using the velocity data through the cycle such that measurements were made on the same anatomical points. ${ }^{12}$ Values of the SR indices $\left(S R_{\text {fiber }}\right.$, $S R_{\text {in-plane }}, S R_{\text {out-plane, }} S R f c$, SR-fiber angle) in the ROIs were taken at the peak of SRfiber in the contraction cycle. Statistical analysis was performed using SPSS (SPSS 23.0, SPSS Inc., Chicago, IL) to test for differences between pre and post ULLS groups (termed time) and three muscle regions as well as potential interaction

\section{Results and Discussion}

The average change in volume of the GM, GL and soleus muscles post-suspension are $-9.6 \%,-11.1 \%$ and $-7.4 \%$ respectively while the average change in MVC is $-32.6 \%$. As seen in earlier studies, ${ }^{3-5}$ the force loss cannot be completely explained by muscle atrophy alone (change in muscle volume). Force produced by the subject during eccentric contractions (averaged over all 7 subjects for pre- and post-suspension separately) is show in Fig 1c; these are an average over 70 contractions/slice, over all 
the slices (5-7/subject) and finally across all subjects. The eccentric contraction begins with the foot in the most plantarflexed phase followed by the pedal exerting force on the foot pushing it to the most dorsiflexed position while the foot is actively contracting (phases 1-11); the pedal then reverses direction while the foot follows the pedal (no force exerted by foot) to the most plantarflexed position (phases 12-24).

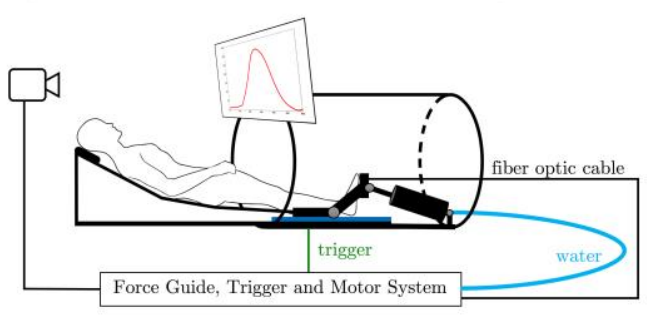

(a)

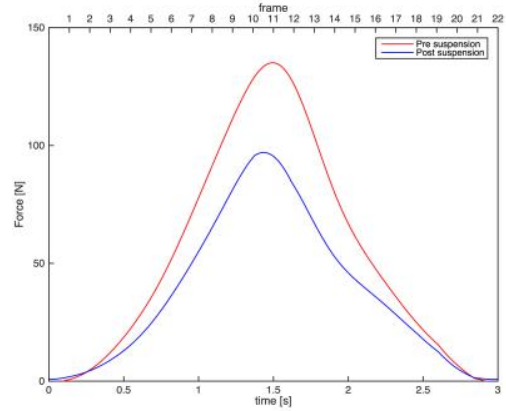

(c)

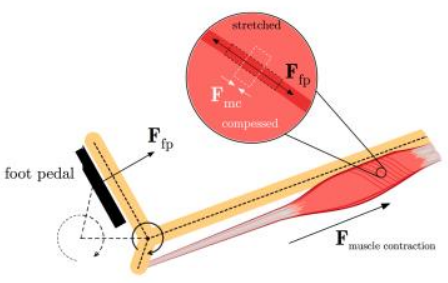

(e)

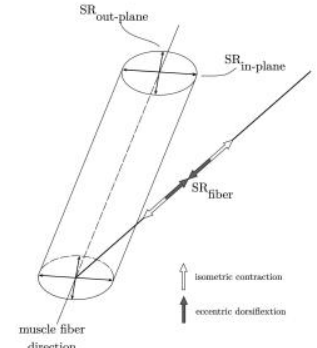

(b)

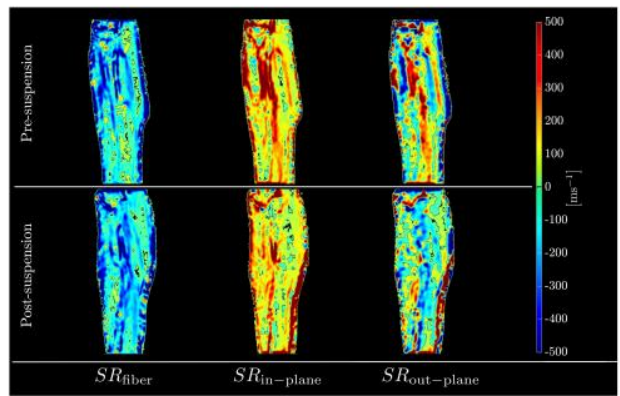

(d)

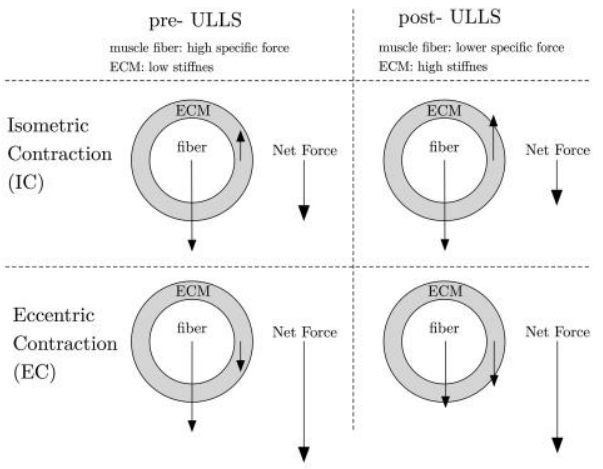

(f)

Fig 1. Foot pedal device and subject foot positioned in the magnet. Trigger from the pressure transducer is used to control the motor (outside the magnet room) that drives the foot pedal, feedback of the generated force profile superposed on the desired force profile is provided (a). Schematic of the muscle fiber and the strain rate components, $S R_{\text {fiber }}$ (closest in orientation to the muscle fiber), $S R_{\text {in-plane }}$ is orthogonal to $S R_{\text {fiber }}$ and in the fiber cross-section, in the imaging plane; $S R_{\text {out }}$ plane is orthogonal to the imaging plane. The angle between $S R_{\text {fiber }}$ and the muscle fiber is denoted as the SR-fiber angle $(\boldsymbol{b})$. Force curves during eccentric contraction (average over all slices and subjects) for pre-(red) and post-suspension (blue) (c). Strain rate maps $\left(S R_{\text {fiber, }} S R_{\text {in-plane }}\right.$,

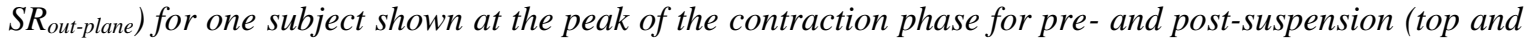
bottom rows). The low values of the SR indices as well as very little change with unloading can be appreciated (d). The free body diagram for eccentric contraction illustrates that the foot pedal causes a dorsiflexion motion while the muscle is actively contracting against the load of the foot pedal. The net deformation measured is the resultant of the contraction due to muscle and the extension due to the dorsiflexion motion from the pedal (e). The effect on force generation due to a stiffer extracellular matrix during isometric and eccentric contraction $(f)$. 
Table 1. Strain rate eigenvalues and the SR-fiber angle for pre-and post-Unilateral Limb Suspension at the same force level as in the post-ULLS during eccentric contraction

\begin{tabular}{|c|c|c|c|c|c|}
\hline \multirow{2}{*}{\multicolumn{2}{|c|}{ Eccentric same force level }} & \multirow[b]{2}{*}{ ULLS } & \multicolumn{3}{|c|}{ region } \\
\hline & & & proximal & middle & distal \\
\hline \multirow{2}{*}{$S R_{\text {fiber }}$} & \multirow{2}{*}[\mathrm{ms}^{-1}]{} & pre & $15158.78 \pm 88.04$ & $221.42 \pm 83.89$ & $308.57 \pm 125.07$ \\
\hline & & post & $118.25 \pm 46.64$ & $147.59 \pm 102.77$ & $214.37 \pm 141.39$ \\
\hline \multirow{2}{*}{$S R_{\text {in-plane }}{ }^{\text {a }}$} & \multirow{2}{*}[\mathrm{ms}^{-1}]{} & pre & $-272.12 \pm 92.35$ & $-310.79 \pm 89.51$ & $-367.94 \pm 69.49$ \\
\hline & & post & $-304.04 \pm 109.76$ & $-380.3 \pm 108.7$ & $-456.56 \pm 181.06$ \\
\hline \multirow{2}{*}{$S R_{\text {out-plane }}$} & \multirow{2}{*}[\mathrm{ms}^{-1}]{} & pre & $113.34 \pm 51.99$ & $89.36 \pm 93.44$ & $59.37 \pm 99.12$ \\
\hline & & post & $185.79 \pm 103.24$ & $232.71 \pm 145.51$ & $242.19 \pm 216.05$ \\
\hline \multirow{2}{*}{\multicolumn{2}{|c|}{ SR-fiber angle }} & pre & $-51.69 \pm 8.6$ & $-45.48 \pm 15.29$ & $-49.97 \pm 11.19$ \\
\hline & & post & $-41.64 \pm 14$ & $-41.17 \pm 8.14$ & $-39.66 \pm 11.25$ \\
\hline
\end{tabular}

a significant interaction time*region

Figure 1d shows the SR eigenvalues and SR-fiber angle corresponding to one subject at baseline (pre-suspension) and immediately after the 4 -week of unloading (postsuspension) during eccentric contraction at the peak of the contraction defined as the peak in $S R_{\text {fiber }}$ (phase/frame: 7). Table 1 shows the eigenvalues in the three muscle regions pre- and post- suspension for eccentric contraction. The eigenvalues during eccentric contraction are smaller than corresponding ones extracted from the isometric contraction by a factor of three. ${ }^{12}$. It should be noted that during the contraction phase (1-11), $S R_{\text {fiber }}$ (defined as the eigenvector closest to the muscle fiber orientation) is the positive eigenvalue while during the relaxation (phases 12-24), $S R_{\mathrm{fiber}}$ is the negative eigenvalue. No significant differences were found in the SR indices for time (pre- and post-) or region. A repeated measures ANOVA on the eccentric data showed significant interactions time * region for SRfiber angle $(\mathrm{F}(2,12)=4.129, \mathrm{P}=0.043)$ and trend towards significance for $S R_{\text {in-plane }}(\mathrm{F}(2,12)=3.597, \mathrm{P}=0.06)$

The lower values of strain rate eigenvalues during eccentric contraction may potentially arise from the fact the muscles are actively contracting while there is lengthening during dorsiflexion caused by external force from the foot pedal. The net result is a smaller extent of tissue deformation due to simultaneous local contraction and elongation. The free body diagram in Figure 1e shows the forces acting on the MG muscle tissue as the foot dorsiflexes under active muscle contraction: force from the foot pedal and the force of contracting muscle act in opposition causing lengthening and contraction respectively (as the final motion is dorsiflexion, the net deformation is lengthening). It should be noted that this is the first in-vivo measurement of the local lengthening along the muscle fiber $\left(S R_{\text {fiber }}\right.$ positive during contraction, Table 1) during an eccentric contraction. In contrast, during isometric contraction $\mathrm{SR}_{\text {fiber }}$ is negative during the contraction part of the cycle (this is anticipated as muscle contraction will result in local shortening). In eccentric contractions, there were no significant differences in any of the SR indices including shear strain between pre- and post-suspension, though the trend of decreased asymmetry of deformation is seen in the post-suspension. This is in contrast to our findings on SR indices during isometric contractions which show significant differences in SR-fiber angles (increase) and in $S R_{\text {in-plane }}$ (decrease). ${ }^{12}$ It is difficult to speculate on the reason for the differences in the change in strain rate indices postsuspension extracted from isometric and eccentric modes of motion. These difference in muscle kinematics on unloading between isometric and eccentric contractions could be related to the fact that a stiffer extra-cellular matrix (post-suspension) may add to the muscle force in the tensile state (eccentric) while it may resist the muscle deformation in the compressive state (isometric) (Fig. 1f). This line of reasoning may also explain larger $S R_{\text {fiber }}$ values seen in the middle and distal regions postsuspension (Table 1). It should be noted that studies have reported that eccentric force is preserved with age while significant decreases in concentric/isometric forces are seen with age. ${ }^{10,11}$ Further, a recent computational model predicted that a stiffer, thicker ECM (as may occur with aging and/or disuse) preserves force during eccentric contraction while a isometric/concentric contraction resulted in a decrease of force. ${ }^{13}$ The observation in the current paper of a lack of strain rate changes seen in eccentric contractions with unloading is in conformance with experimental observations and computational predictions of force being preserved in eccentric contractions with aging.

\section{List of acronyms}

$3 \mathrm{D}^{\text {al }}$ - three-dimensional (

DENSE - displacement encoded imaging

ECM - Extra-cellular Matrix

GL - Lateral Gastrocnemius

GM - Medial Gastrocnemius

MRI - Magnetic Resonance Imaging

MVC - maximum voluntary contraction

SPAMM - Spatial Modulation of Magnetization 
SR - Strain Rate

ULLS - Unilateral Limb Suspension

VE-PC - Velocity encoded phase contrast imaging

\section{Authors contributions}

US: manuscript writing; VM: data collection, analysis, figures, manuscript revision; RC: data collection, manuscript revision; $\mathrm{MN}$ : manuscript revision; SS: data collection, manuscript revision

\section{Acknowledgments}

Part of this paper was an e-poster presentation at the 24th Annual Meeting of the International Society of Magnetic Resonance in Medicine, 7-13 May 2016, Singapore.

\section{Funding}

This work was supported by the National Institute of Arthritis and Musculoskeletal and Skin Diseases Grant R01AG056999-01

\section{Conflict of Interest}

The authors declare no potential conflict of interests.

\section{Ethical Publication Statement}

We confirm that we have read the Journal's position on issues involved in ethical publication and affirm that this report is consistent with those guidelines.

\section{Corresponding Author}

Shantanu Sinha, Radiology Imaging Laboratory, University of California at San Diego, 3510, Dunhill St., San Diego CA 92121 USA. Phone: 858-534-2004

Fax: 858-534-6046

ORCID iD: 0000-0003-0796-2694

Email: shsinha@ucsd.edu

E-mail and ORCID iD of co-authors

Usha Sinha: usinha@sdsu.edu

ORCID iD: 0000-0002-1160-2483

Vadim Malis:vmalis@ucsd.edu

ORCID iD: 0000-0002-0426-1457

RobertCsapo: robert.csapo@umit.at

ORCID iD: 000-0003-3571-2799.

Marco Narici: marco.narici@unipd.it

ORCID iD: 0000-0003-0167-1845

\section{References}

1. Bodine SC. Disuse-induced muscle wasting. Int J Biochem Cell Biol 2013;45:2200-8.

2. Bongers KS, Fox DK, Kunkel SD, et. al. Spermine oxidase maintains basal skeletal muscle gene expression and fiber size and is strongly repressed by conditions that cause skeletal muscle atrophy. Am J Physiol Endocrinol Metab 2015;308:E14458.

3. Thomason DB, Booth FW. Atrophy of the soleus muscle by hindlimb unweighting. J Appl Physiol 1990;68:1-12.

4. de Boer MD, Maganaris CN, Seynnes OR, et. al. Time course of muscular, neural and tendinous adaptations to 23 day unilateral lower-limb suspension in young men. J Physiol 2007;583:1079-91.

5. de Boer MD, Selby A, Smith K, et. al. The temporal responses of protein synthesis, gene expression and cell signalling in human quadriceps muscle and patellar tendon to disuse. J Physiol 2007;585:24151.

6. Lieber RL, Ward SR. Cellular mechanisms of tissue fibrosis. 4. Structural and functional consequences of skeletal muscle fibrosis. Am J Physiol Cell Physiol 2013;305:C241-52.

7. Ramaswamy KS, Palmer ML, van der Meulen JH, et. al. Lateral transmission of force is impaired in skeletal muscles of dystrophic mice and very old rats. J Physiol 2011;589:1195-208.

8. Sharafi B, Blemker SS. A mathematical model of force transmission from intrafascicularly terminating muscle fibers. Journal of Biomechanics 2011;44:2031-9.

9. Bilston LE, Bolsterlee B, Nordez A, Sinha S. Contemporary image-based methods for measuring passive mechanical properties of skeletal muscles in vivo. J Appl Physiol 2019;126:1454-64.

10. Barber LA, Barrett RS, Gillett JG, et al. Neuromechanical properties of the triceps surae in young and older adults. Exp Gerontol 2013;48:1147-55.

11. Roig M, MacIntyre DL, Eng JJ, Reid WD. Preservation of eccentric strength in older adults: Evidence, mechanisms and implications for training and rehabilitation. Exp Gerontol 2010;45:400-9.

12. Malis V, Sinha U, Csapo R, et al. Relationship of changes in strain rate indices estimated from velocity-encoded MR imaging to loss of muscle force following disuse atrophy. Magn Reson Med 2018;79:912-22.

13. Zhang Y, Chen J-S, He Q, He X, et al. Microstructural analysis of skeletal muscle force generation during aging. - PubMed - NCBI. Int $\mathbf{J}$ Numer Meth Biomed Engng 2019;52:B125.

Submitted: March 2, 2020 Accepted for publication: March 2, 2020 